\title{
La exclusión entre pares: sus implicaciones en la formación de los y las estudiantes
}

\section{Exclusion among peers: its implications for the students' education}

\section{DOI: 10.32870/dse.v0i19.489}

\author{
Lidia Isabel Castellanos Pierra* \\ Federico Zayas Pérez ${ }^{* *}$
}

\begin{abstract}
Resumen
El presente documento tiene como objetivo atender a las formas de exclusión que se producen en la escuela, a través de la interacción entre estudiantes del nivel medio superior, tanto en el espacio áulico como fuera de éste. Interesa comprender en qué medida la exclusión que se manifiesta por medio de las dinámicas relacionales entre pares se acompaña de implicaciones para la formación de estos alumnos.

Como referentes teóricos, se acude al concepto de intersubjetividad desde la perspectiva fenomenológica, así como a presupuestos del interaccionismo simbólico y a la teoría del reconocimiento de Honneth, los cuales ayudan a articular los conceptos centrales de exclusión-interacción-formación.

El trabajo se desarrolló a partir de la revisión bibliográfica que permitió ubicar diversos estudios e investigaciones relacionados con la temática, conocer los principales enfoques y abordajes emprendidos, tanto en el ámbito internacional como nacional.

Los resultados de la indagación realizada revelan la actualidad de la problemática, así como la necesidad de atender a las características de las relaciones que establecen entre sí los y las estudiantes, sobre todo si se considera la importancia de las mismas para el logro efectivo de los saberes curriculares y el desarrollo satisfactorio de los procesos identitarios individuales y colectivos.
\end{abstract}

Palabras clave: exclusión entre pares - interacción - formación.

\begin{abstract}
This paper aims to call attention to the practices of exclusion that take place in the school through the interaction among high school students, both in the classroom and outside of it. Our goal is to understand to what extent the exclusion that manifests itself through relational dynamics among peers is accompanied by implications for the formation of these students.
\end{abstract}

\footnotetext{
* Máster en Ciencias de la Educación Superior por la Universidad de Oriente, Cuba. Doctorante del Programa de Doctorado en Ciencias Sociales de El Colegio de Sonora, generación 2016-2019. Líneas de investigación: sociología de la educación, educación comparada, teorías de la educación. México. lidiaisabelcp@yahoo.es

** Doctor en Educación Internacional, profesor de la Universidad de Sonora. Línea de investigación: teoría social en psicología, psicología social, sociología de la educación, políticas educativas, evaluación psicológica y análisis, diseño y evaluación del currículo, teoría de la educación y metodología cualitativa. México.fzayas@psicom.uson.mx
} 
As theoretical referents, we turn to the concept of intersubjectivity from a phenomenological perspective, as well as to the presuppositions of symbolic interactionism and Honneth's recognition theory, which help to articulate the central concepts of exclusion, students' interaction and education.

The research work consisted of bibliographic review that allowed us to find different studies and research related to the subject, as well as to learn about the main approaches adopted both in our country and abroad.

The results reveal the current situation of the problem, as well as the need to attend to the characteristics of the relationships established among the students, especially considering the importance of peer relationships for the effective achievement of the curricular goals and the satisfactory development of individual and collective identity processes.

Key words: peers exclusion - interpersonal relationships - secondary schooling

\section{Introducción}

Al hablar de exclusión se alude a un concepto complejo, a un fenómeno que se manifiesta en diferentes ámbitos de la vida social e involucra factores diversos e interrelacionados. Entre estos, a la estructura socioeconómica, al aparato institucional y organizacional de carácter político e ideológico y a los procesos sociorrelacionales que atañen a los colectivos, los grupos y los individuos. Al respecto, encontramos diversas perspectivas de análisis de la exclusión cuyo interés se centra ya sea en los mecanismos de desprotección social, en la discriminación activa hacia ciertos grupos estigmatizados o bien, en los procesos de relaciones entre los individuos y grupos (Pluma, 2011). En cualquiera de estas perspectivas, se asume que la exclusión lleva implícita la vulneración de los derechos de un colectivo, de una comunidad o de un individuo, y se manifiesta como privación de derechos, negación de oportunidades; a la vez que la misma se entrelaza con actitudes y prácticas discriminatorias al conducir al apartamiento deliberado, a la desafiliación y al trato desigual entre los seres humanos.

El tema de la exclusión social no se reduce solo a la pobreza y a las desigualdades en la pirámide social, sino a la medida en que se tiene o no un lugar en la sociedad en la que es posible participar y beneficiarse de esta o, por el contrario, ser rechazado e ignorado como resultado de la misma dinámica social (Bel, 2002). Siendo así, "una sociedad excluyente no es únicamente una sociedad donde hay pobres, es una sociedad donde desaparecen las posibilidades de concretar derechos fundamentales universales" (Rodríguez, 2007: 99).

Los procesos escolares no están exentos de este fenómeno. Está presente tanto en sus extensas manifestaciones estructurales como en la exclusión de amplios grupos sociales, por clase, espacios geográficos, grupos étnicos o géneros, hasta en las finas y apenas perceptibles expresiones de exclusión en la interacción entre los sujetos escolares: rechazar e ignorar o ser rechazado e ignorado mediante el tono de voz, el sesgo de la mirada, el gesto de las manos o

Diólo@os sobre Educación año 10 | número 19 | julio-diciembre 2019 | ISSN 2007-2171 
el silencio. En este trabajo se pretende atender esta última dimensión del fenómeno, por ello se enfoca en especial hacia la interacción entre los y las estudiantes y, por su interés para la educación escolar, se busca comprender lo que esa exclusión implica en los cambios en las conductas, los conocimientos y los sentimientos de los alumnos.

\section{Las relaciones entre la exclusión y la educación}

La exclusión social ha sido analizada en una estrecha relación con la exclusión educativa, en tanto la primera refiere una condición que en muchos casos deriva de la falta de participación en los procesos educativos (Ramírez, 2014). La educación adquiere un peso relevante como factor de integración social al proveer a las personas de los recursos y competencias necesarios para desempeñarse en la sociedad contemporánea altamente tecnificada y donde el conocimiento se torna indispensable, mas no suficiente. Por el contrario, la falta de escolaridad suele aparejarse con situaciones de marginación, precariedad y exclusión social, lo cual incrementa las dificultades para que los individuos o grupos puedan desarrollarse, tanto en el orden profesional como en el nivel personal y social.

La exclusión educativa no se reduce únicamente a la imposibilidad de acceder a una educación escolar, sino que tiene múltiples expresiones, las cuales forman parte del funcionamiento y la lógica de los propios sistemas educativos. Al respecto, Echeita y Sandoval (2002: 33) comentan que:

los sistemas educativos, a través de mecanismos unas veces "explícitos" (por ejemplo, la segregación de los alumnos con discapacidad en centros especiales) y otras "implícitos u ocultos" (como la sobrevaloración de las capacidades de tipo intelectual, las prácticas de selección y evaluación del alumnado o la existencia de un currículo rígido y centralizado) han sido y en muchos casos siguen siéndolo, los "primeros" en generar fuertes contingentes de alumnos en riesgo de exclusión social.

Por consiguiente, ser excluido no depende únicamente de estar dentro o fuera de la escuela. En el caso de Latinoamérica, y en México en particular, si bien se han logrado importantes avances en la consecución del acceso universal a la escuela básica, lo que favorece el derecho de igualdad de oportunidades en este sentido, se ha visto cómo en esos esfuerzos por alcanzar la anhelada cobertura total, la masificación ha arrastrado consigo la reproducción de las desigualdades sociales en el interior del sistema escolar, de las escuelas y las aulas. En el plano del sistema escolar, se identifican integraciones diferenciadas pues son las mejores escuelas las que reciben a los alumnos con mayor capital social, cultural y económico; mientras que las escuelas con mayores dificultades en infraestructura y recursos humanos acogen a un alumnado con evidentes desventajas sociales y educativas, y con mayor riesgo de experimentar el fracaso escolar. En una dimensión escolar, también se expresan desigualdades socioeconómicas y culturales 
que se revelan en inclusiones desiguales. Esto da paso a la "conformación de distintos mundos escolares, cada uno de ellos socialmente homogéneos y recíprocamente distantes y desconocidos" (Saraví, 2015: 90). Por ello, los jóvenes que se encuentran en condiciones de mayor vulnerabilidad, también son los más excluidos.

\section{La exclusión como forma de interacción entre pares estudiantes en la escuela}

Por otra parte, la exclusión se manifiesta en el ámbito de las dinámicas relacionales en la escuela. Ella constituye una de las frecuentes expresiones de violencia en las escuelas, relacionada con otros tipos de maltrato como el verbal, la agresión física y el maltrato psicológico (Arellano, Chirinos, López y Sánchez, 2007; Estrada; Zárate e Izquierdo, 2016; Valadez, 2008).

Sánchez (2016) señala que, en México, los primeros esfuerzos académicos vinculados a temas como la disciplina, el reglamento escolar, los patrones de comportamiento, etc., se llevaron a cabo durante los años noventa, y en su mayor parte eran publicaciones coordinadas por el Consejo Mexicano de Investigación Educativa (COMIE). Gradualmente se fue consolidando una línea de investigación centrada en el tema del acoso escolar, y ya para la década de los 2000 se publicó el primer estado de conocimiento que reunía los principales avances arrojados por la discusión conceptual y el trabajo empírico acerca de lo producido en diez años en cuanto a convivencia, disciplina y violencia en las escuelas.

Recientemente, en lo que respecta al análisis de la exclusión y la violencia en la educación, habría que destacar los trabajos realizados por investigadores y egresados de la Universidad Autónoma de San Luis Potosí (Portillo, Acosta, Sánchez, Ruiz, Ávila y Rodríguez, coordinados por Solís, 2016), quienes a través de diferentes disciplinas como la historia, la antropología o la psicología social, abordan procesos y situaciones donde se manifiestan las relaciones entre el campo educativo y la exclusión, la discriminación y la violencia, que a la vez develan los mecanismos que generan y permiten múltiples expresiones excluyentes y discriminatorias en los centros escolares.

De igual forma, ha de mencionarse el trabajo de Lira, Sandoval y Aguilar (2016) cuya propuesta se orienta hacia la comprensión de la violencia escolar a partir de la delimitación de una serie de estructuras para la convivencia, identificadas por los autores. Una de estas estructuras se refiere precisamente a la estructura de la interacción para la inclusión o exclusión. En ese marco, la exclusión ha sido entendida sobre todo como un problema de comunicación que se manifiesta por medio de conflictos derivados de la confusión de sentidos, pero también cuando existe un ambiente invalidante o de negación del otro. El análisis muestra cómo desde la propia institución escolar se establece la estructura de organización para la convivencia en la escuela, a partir de la cual se construyen y aceptan las normas por parte de sus miembros, se generan formas de comunicación incluyentes o no, así como modos para afrontar y resolver los problemas escolares diarios. 
En sentido general, los estudios desarrollados revelan la existencia de un pluralismo conceptual y metodológico acorde a la complejidad de la problemática (Furlán, 2012). Habría que destacar el abandono paulatino de los modelos unicausales y las posturas ceñidas a un único paradigma, para transitar hacia abordajes multi e interdisciplinarios. Se acude a modelos como el ecológico-sistémico, que toman en cuenta los aspectos estructurales, institucionales y psicológicos. Desde este tipo de modelos se visualiza a los y las adolescentes y a las diferentes estructuras en las que se encuentran inmersos: familia, escuela, barrio, comunidad, organizaciones, "todas ellas incorporadas en un amplio contexto social que incluye a las políticas públicas, la cultura y el medio ambiente físico" (Valadez, 2008: 30).

Al mismo tiempo que se comprenden las interrelaciones entre las dimensiones estructurales y la dinámica interna de los centros educativos, se mantiene el interés por emprender enfoques de carácter microsociológico para atender a esas violencias leves o microviolencias, mucho más sutiles que, no obstante su recurrencia, suelen pasar desapercibidas a pesar de tener un peso importante en la vida diaria de los agentes escolares (Furlán, 2012). Precisamente por su presencia continua, son prácticas que se han naturalizado, es decir, se perciben inherentes a la naturaleza humana y se asumen como parte inevitable de la convivencia escolar y social (Solís, 2017). Al naturalizarse, la violencia, la exclusión y la discriminación se tornan invisibles, a la vez que se desconocen los condicionamientos sociohistóricos y culturales que las gestaron.

Los resultados de las investigaciones sobre los conflictos vividos en la escuela invitan a reconsiderar la imagen de esta como un lugar seguro e ideal para la integración social; en la medida en que la violencia se instala y se hace palpable cada vez con mayor fuerza en los recintos escolares, teniendo en cuenta que no se trata solo de hechos aislados, sino de un ambiente, un clima, una atmósfera, que intenta anular al otro sujeto de la interacción de diversas maneras (Velázquez, 2005).

De acuerdo con los resultados del Programa Internacional de Evaluación de los alumnos (PISA) del año 2015 (citados por la OCDE, 2017), en lo que concierne a la vida social en la escuela, en México, $25 \%$ de los alumnos se siente marginado en el colegio (la media de la OCDE es de $17 \%$ ) y $21 \%$ se siente solo (la media de la OCDE es de $15 \%$ ). Estas cifras manifiestan las dificultades que enfrentan muchos estudiantes para relacionarse con sus compañeros en la escuela. Aunque el fenómeno de la exclusión está presente en todos los niveles escolares, en adelante se enfocará hacia la educación media superior y, de manera más específica a los procesos de exclusión entre pares. Al respecto, los resultados de la Encuesta Nacional sobre Exclusión, Intolerancia y Violencia en las Escuelas de Educación Media Superior (SEP, 2009) indicaron que en México, $42.3 \%$ de los estudiantes hombres y $41.4 \%$ de las mujeres dijo haber sido ignorado. También revelaron que, tras ser segregados, $30.5 \%$ de las mujeres y $19 \%$ de los hombres pensaron que era preferible morir. En tanto, quienes expresaron haber rechazado constituían $36.5 \%$ entre los hombres y $35.3 \%$ en el caso de las mujeres. 
En la introducción de los resultados de la Encuesta Nacional sobre Discriminación en México del año 2010, específicamente aquellos relacionados con los y las jóvenes, se reconocía que:

Falta mucho por trabajar en la formación de la conciencia crítica, la eliminación de prejuicios y la construcción de nuevos escenarios que garanticen la inclusión. No sorprende que haya jóvenes que no desean convivir con personas que tienen preferencias sexuales distintas a la heterosexual, con personas que viven con $\mathrm{VIH} /$ sida, con indígenas y personas con discapacidad. La necesidad de pertenencia e identidad se confunde con la división y la segregación (Castellanos, en Instituto Mexicano de la Juventud-Consejo Nacional para Prevenir la Discriminación, 2011: 13).

Por otra parte, es oportuno señalar que el modelo educativo vigente para el Bachillerato General en México, desarrollado a partir de la Reforma Integral para la Educación Media Superior (RIEMS) del año 2008 (SEP, 2016), se fundamenta en presupuestos teórico-epistemológicos del constructivismo sociocultural. Desde este, el aprendizaje se concibe como"un proceso esencialmente social e interactivo, que consiste en una auténtica apropiación de los recursos culturales gracias a la participación de la/el aprendiz en una actividad conjunta con otros" (SEP, 2016: 21).

El modelo educativo del Bachillerato General acoge también un enfoque intercultural en el diseño y contenidos del plan de estudios, orientado fundamentalmente a promover y desarrollar prácticas ciudadanas sustentadas en valores cívicos como la apertura al diálogo, la tolerancia, el respeto a la diversidad y a la diferencia; todos ellos indispensables para atender problemáticas específicas que en la actualidad forman parte de las prácticas cotidianas de las y los jóvenes mexicanos (SEP, 2016). Se pretende que estos elementos permeen el currículum y se concreticen en el perfil de egreso del bachiller, a través del desarrollo de competencias genéricas que trasciendan el contexto escolar y se tornen relevantes a lo largo de toda la vida, por tratarse de aspectos esenciales no solo para la formación profesional, sino para la formación integral y humanista de los y las estudiantes.

Sin embargo, más allá de la retórica oficial y de los documentos que rigen el proyecto institucional, en la escuela se manifiestan paralelamente creencias, ideologías, modos de relación diversos y no necesariamente convergentes con las pretensiones educativas expuestas en el modelo curricular vigente. Estos elementos engrosan el currículum oculto, aquel que se enseña y se aprende a la par de los contenidos explícitos de los programas educativos y que puede ser tanto o más importante que estos para la reproducción social, para la formación de las personas y las sociedades (Kaplún, 2003).

En paralelo con el quehacer académico y las actividades de instrucción propias de la escuela, ese currículum constituye también un espacio de vida juvenil (Weiss, 2014), donde los alumnos intercambian conocimientos diversos sobre la vida, puntos de vista, valores, creencias, hábitos, establecen relaciones afectivas, crean lazos de amistad, se enamoran, pero también

Diólo@os

sobre Educación

Sestion año 10 | número 19 | julio-diciembre 2019 | ISSN 2007-2171 
viven conflictos y enemistades; participan así de múltiples y variadas experiencias. Al mismo tiempo, los y las estudiantes participan en procesos de socialización, aprenden reglas y valores establecidos por los adultos, pero sobre todo, por los grupos de pares a los que pertenecen. Por estas razones, los estudiantes se ven en la necesidad de desarrollar habilidades para aprender a comportarse según la normatividad específica de los distintos espacios y contextos por los que transitan, ámbitos de interacción diversos que como tales exigen distintos modos de relación. En este sentido, las normas y valores del grupo de pares en la escuela manifiestan generalmente una distancia con respecto a aquellas que rigen el mundo de los adultos (profesores, autoridades, padres) y mientras mayor sea el contraste con aquel, mucho mejor (Harris, 1995).

En la medida en que los alumnos interactúan, se genera "el microsistema de los iguales en el que se gesta la cultura del grupo compuesta por normas, ritos, convenciones, creencias y hábitos de comportamiento; misma que marca las pautas de su comportamiento social" (Valadez, 2008: 28). En este microsistema, muchas veces se desarrolla una especie de competencia grupal caracterizada por relaciones de dominio-sumisión entre los miembros del grupo, o entre subgrupos, modos de relación que pueden producir un desequilibrio entre el estatus social de los miembros y poner en riesgo el vínculo de la reciprocidad (Valadez, 2008). Son relaciones que llevan al reto, al desafío, de las cuales es difícil escapar. En ellas, el prestigio y el poder aumentan de forma proporcional a la transgresión de las reglas escolares, "relaciones afectivas más que racionales, que llevan a la integración de algunos y al rechazo total o parcial de otros" (Tello, 2005: 1173).

La exclusión forma parte de estas dinámicas relacionales entre los y las estudiantes, cuyas manifestaciones más comunes suelen ser la indiferencia, el rechazo, el apartamiento, la negación del reconocimiento del otro, a quien se le impide participar, es decir, formar y tomar parte, tener voz y ser escuchado. Muchas de estas acciones responden a un repertorio de prejuicios y estereotipos que llevan a la discriminación, sobre todo de aquellos que son considerados como diferentes porque no se adaptan al perfil de una identidad hegemónica, o porque simplemente no encajan en los estándares normalizadores del grupo al que pertenecen. Con ello se vulneran derechos humanos fundamentales como el derecho a la equidad entre las personas, derechos individuales $y$, muy especialmente, el derecho a la igual consideración y respeto, que son base del principio antidiscriminatorio (Cisneros y Cisterna, 2007). Es común, además, que estas prácticas de exclusión se acompañen de ciertas formas de menosprecio, como la humillación física y la devaloración social. Al ser menospreciado, "el individuo sufre la consecuencia de que no puede recurrir a través del fenómeno positivo de la apreciación social, a su propia autovaloración [...], se ve inducido y presionado a devaluar su forma de vida propia y a sufrir una pérdida de autoestima" (Arias, 2015). Se vulnera así la dignidad y la integridad de la persona. Esta devaluación del autoconcepto, producto del rechazo y la negación experimentada, dificulta y distorsiona la comprensión de sí mismo y de los otros, atentando por consiguiente contra los procesos de formación. 
Se verá en adelante cómo estas interacciones excluyentes inciden en la formación de quienes en ellas participan.

\section{El yo y el otro, el reconocimiento, la socialización y la subjetivación en la interacción entre pares: sus implicaciones en la formación de los y las estudiantes}

Vale recordar que los procesos formativos no se restringen a la escuela. Estos se extienden y realizan en los múltiples y variados contextos donde se desarrolla la vida social: la familia, el barrio, la iglesia, el trabajo, los ambientes lúdicos y muchos más.

El concepto de formación va más allá de la adquisición de conocimientos y habilidades necesarias para el futuro desempeño técnico-profesional; rebasa la simple instrucción y alcanza un sentido trascendental y un carácter mucho más abarcador, en la constitución amplia e integral del sujeto. Esto lleva a considerar aspectos axiológicos, en sintonía con los diversos saberes preconizados para el hombre y la mujer del siglo XXI, que han de prepararse no solo en el saber y el saber hacer, sino, también, en el saber ser y el saber convivir (Delors, 1994).

En el ámbito educativo, resulta pertinente diferenciar aquella formación que compete a los procesos desarrollados las formas de enseñanza y guiados por una lógica de normalización y homologación de las diferencias, de otro tipo de formación (bildung), que rehúye todo intento de moldeamiento, y se expresa "en el movimiento por la acción del formando, que se concreta en esas objetivaciones y en la recuperación reflexiva y crítica de su experiencia, el esfuerzo de subjetivación" (Yurén, 2012: 82). Se trata de una formación que nace, no de una necesidad impuesta desde el exterior sino desde los anhelos más íntimos del sujeto, de sus constantes cuestionamientos, de la duda perpetua, por lo cual nunca se completa, ni finaliza.

La formación puede ser definida como un proceso de construcción de significados, sentidos e identidades o, como "la configuración individual y social de los sujetos a través de los significados culturales puestos en juego por la escuela, presionados éstos por los significados propios de los otros contextos del sujeto y por la construcción de todos ellos realizada por cada individuo" (Zayas y Rodríguez, 2010: 15).

La formación compromete así a cada persona en los procesos de internalización de los significados y en los procesos de subjetivación. Entendiendo a estos últimos como procesos reflexivos de comprensión de sí mismo y de los otros en el mundo real. Pero a la vez, la formación reviste un carácter social pues los significados son construidos, recreados y compartidos en la intersubjetividad, mediante la interacción con el otro en el mundo que les rodea. Es precisamente a través de los significados compartidos por medio del lenguaje en la interacción, que resulta posible la comunicación y el entendimiento entre los seres humanos.

La interacción demanda siempre la comunicación con otro distinto a uno mismo (Rizo, 2006) y presupone, además del intercambio de sentidos, la reciprocidad, la influencia mutua y

Diálo@os sobre Educación 
la creación de un espacio sociopsicológico común, donde la intersubjetividad permite que "estas conciencias se construyan y accedan al sentimiento de su identidad" (Marc y Picard 1992: 60). Desde esta perspectiva, cada individuo solo puede conocerse a sí mismo a partir de la relación con los demás y sobre la base del reconocimiento del otro.

Este proceso de reconocimiento recíproco y de reconocimiento de sí mismo, entraña ciertas tensiones entre el Ego y el Alter, debido al tránsito por etapas de reconciliación y conflicto; las primeras, caracterizadas por la conexión con el otro al saberse reconocido por este, y las etapas de conflicto, definidas por la autoafirmación, en la medida en que se posee una identidad personal única e irremplazable que se contrapone a la de aquel (Honneth, 1997).

En los presupuestos del interaccionismo simbólico se subraya el carácter social de los procesos de significación, de construcción de sentidos y de constitución de la identidad, todos implícitos en la formación; por cuanto la significación surge y reside dentro del campo de la relación. "El individuo se experimenta a sí mismo como tal, no directamente, sino solo indirectamente, desde los puntos de vista particulares de los otros miembros individuales del mismo grupo social" (Mead, 1982: 169).

Siendo así, el individuo solo puede llegar a una conciencia de su identidad al mirarse desde fuera, colocándose en la perspectiva de los otros con quienes interactúa. En la interacción con el otro, el individuo (el yo-otro) orienta, o no, su vida; le da sentido a su existencia.

Desde estos posicionamientos teóricos, es posible comprender la importancia de la dimensión relacional para el desarrollo del proceso formativo. Precisamente porque:

La formación es estimulada por el reconocimiento de los otros y un sujeto no reconocido y tratado como objeto difícilmente entra en un proceso de formación [...]. El sujeto que se forma demanda de los otros con quienes se forma el reconocimiento como sujeto. Por eso, un ambiente intersubjetivo favorece la formación (Yurén, 2012: 85).

En sentido contrario, toda ruptura o quiebre relacional, cualquier práctica de devaluación del otro, obstruye la posibilidad de crear un vínculo intersubjetivo, necesario para el reconocimiento y la autorrealización.

Las fracturas relacionales se producen a través de interacciones excluyentes que no se reducen al aislamiento físico del otro sujeto de interacción. La exclusión se presenta también cuando los miembros de una organización o grupo impiden a una persona participar. De igual forma, puede verificarse la exclusión cuando se utilizan señalamientos peyorativos, estigmas o por ruidos y conflictos en la comunicación (Lira, Sandoval y Aguilar, 2016).

Estas situaciones de exclusión y rechazo escolar entre pares pueden resultar más o menos evidentes, siendo común que se minimice su gravedad al considerarlas como inevitables, como problemas que deben aprender a resolver entre ellos, sin que los adultos intervengan. De for- 
ma contraria a lo imaginado, el efecto de estas prácticas suele ser profundo en la formación de las personas, y lacera los niveles de autoestima del individuo, el autoconcepto, la identidad. Además de afectar el desarrollo de habilidades sociales, así como el aprendizaje de actitudes y valores (Estévez, Martínez y Jiménez, 2009).

Desde el punto de vista académico, hay que recordar el papel de los pares como mediadores indispensables para lograr avances en el desarrollo cognitivo. Las interacciones entre los estudiantes pueden resultar altamente favorecedoras en este sentido, al permitir la comparación de puntos de vista diversos para la resolución de ejercicios o tareas conjuntas; o ante la exposición y fundamentación de las perspectivas individuales, así como para la coordinación y control de roles y el ofrecimiento y recepción de ayuda mutua (Coll et al., 1999, citado en Meza, 2010).

En un esfuerzo por rehuir del aislamiento y el rechazo por parte de sus compañeros, y al intentar ser integrados, algunos estudiantes optan por un acoplamiento incondicional con los estándares del grupo, aún en contra de la adecuación de normas o de valores organizacionales o sociales. En este orden de ideas, Velázquez (2005) describe casos de estudiantes preparatorianos quienes relatan "cómo los otros chicos no les querían y hasta les odiaban por ser 'buenos estudiantes', como consecuencia de esto, deciden cambiar y dejar de ser 'buenos alumnos' como una estrategia vital defensiva, frente a un ambiente que les es hostil" (2005: 747). Se revelan así procesos de heterosubjetivación (Wieviorka, 2011), donde intervienen los demás, pero no en el sentido de una coformación favorecedora, sino en un sentido coercitivo, de imposición y moldeamiento, que da paso a una identidad construida desde y para los otros. También es posible encontrar algunos estudiantes que escogen el apartamiento voluntario y renuncian a lo social para afirmarse a sí mismos con más fuerza, cayendo en la autoexclusión (Wieviorka, 2011; Yurén, 2012).

En su experiencia escolar, los adolescentes jóvenes viven así una continua tensión entre los intentos de socializar y de subjetivarse. Mientras la socialización se centra en la interiorización y el aprendizaje de normas intergeneracionales e intrageneracionales mediante la interacción, la subjetivación conduce hacia una emancipación de las ideas y normas dominantes para favorecer el desarrollo autónomo, el conocimiento emocional de sí mismo y la capacidad de reflexionar (Weiss, 2012). Ambos procesos participan en la formación de los sujetos y requieren la presencia de los otros, necesarios tanto para facilitar el conocimiento y la apropiación de la cultura, como para generar experiencias que hagan posible la autotransformación.

Este marco conceptual permite comprender las implicaciones de las interacciones excluyentes en la formación de los y las estudiantes.

\section{Convivir en la diversidad}

Cuando se indaga en los elementos propiciadores de las formas de exclusión y rechazo entre los pares, se descubren múltiples factores que pueden dar lugar a actitudes y comportamientos ex-

Diálo@os sobre Educación año 10 | número 19 | julio-diciembre 2019 | ISSN 2007-2171 
cluyentes en el estudiantado. Como se ha señalado en páginas precedentes, entre los múltiples motivos sobresale el hecho de ser percibido como "diferente", ya sea en un sentido que puede llevar a ser subestimado o a ser sobrevalorado por el agresor. En cualquier caso, "la diferencia" contribuye a convertir a su portador en un objetivo potencial del maltrato ejercido por los pares en la escuela.

Pero, ¿cómo identificar lo diverso, lo diferente? ¿Diverso y diferente con respecto a qué o a quién? ¿Diverso y diferente para bien o para mal? En este sentido, resulta necesario reflexionar acerca del carácter social que acompaña al concepto de diversidad, por cuanto la diversidad se construye a partir de ciertos parámetros propios de los distintos sistemas sociales, que sirven como norma para establecer qué o quiénes son diferentes. De este modo "se es diverso a partir de lo que se observa y se construye considerando criterios de tipificación social [...] que por tanto colocan a la diversidad y a la diferencia dentro de un sistema [...] tomando como referencia a los otros sistemas sociales" (Ramos, 2012: 94). Los criterios utilizados para establecer lo que es diverso, pueden contemplar aspectos de carácter social como la etnia, el género, la lengua, la religión, la posición socioeconómica, etc.; pero también factores ligados a las características funcionales de los individuos, vinculados a sus estilos cognitivos, a aspectos motivacionales, a ciertos rasgos temperamentales, a modos de actuar y de relacionarse, entre otros. En cualquier caso, son criterios que poseen un carácter sociohistórico y se encuentran circunscritos a un marco cultural de interpretación, el cual condiciona la forma de comprender el mundo, de entender qué es lo diverso y de otorgarle un valor. Subyacen en ello modos de conocer e interpretar la realidad social, aprehendidos e interiorizados desde la infancia, mediante los procesos de socialización, donde la familia y la escuela desempeñan un rol fundamental. Procesos por medio de los cuales se aprende a clasificar y a diferenciar a las personas y a los grupos, según estereotipos y categorías sociales no exentos de atribuciones morales y juicios de valor.

Aunque el grupo escolar se erige como un pequeño sistema social, con su propia cultura e identidad que le singulariza y distingue, no ha de olvidarse que al mismo tiempo se encuentra en una relación circular con el ambiente externo, de manera que ambiente y grupo se influyen y modifican recíprocamente. El grupo desarrolla de este modo comportamientos que le permiten adaptarse y sobrevivir en el ambiente. Por consiguiente, la comprensión de los modos de relación que desarrollan los estudiantes en sus grupos de clase y en los grupos informales que constituyen en la escuela, invitan necesariamente a atender a lo que ocurre fuera de la escuela, a los flujos culturales hegemónicos y emergentes que llegan desde otros espacios mayores de la estructura social, a través de los contenidos que trasmiten los medios de comunicación, de las relaciones que establecen con sus amigos en el barrio, de los patrones de comportamiento que viven en la familia y de los propios modos de relación que caracterizan a la cultura y a la organización de la institución escolar, los cuales aprehenden en su cotidianidad. Se trata por tanto de reconocer el carácter sistémico y dinámico de las interacciones. 
En este contexto, y en relación con la percepción que los propios estudiantes tienen acerca de las prácticas de exclusión, discriminación y violencia que viven en la escuela, Solís (2017) señala:

Este conocimiento es experiencial, adquirido en los espacios en los cuales, ellas y ellos transitan de manera concreta, pero también es simbólico y desborda lo local y remite a un espacio social amplio en el cual ubican procesos de exclusión. En su cotidianidad, deambular en la calle, estar en la familia e incluso en las formas de vestir, experimentan, perciben y conocen la exclusión social. La exclusión se percibe en las interacciones, relaciones, procesos que [...] forman parte de la vida cotidiana de los y las estudiantes (Solís, 2017: 66).

¿Qué hacer al respecto? Lo más importante es reconocer la articulación de los diferentes niveles contextuales de la exclusión: sociedad-barrio-escuela-aula, y la importancia de involucrar en las acciones a todos los miembros de la comunidad educativa: estudiantes, profesorado, autoridades, padres de familia, para contrarrestarla.

Si bien el sistema educativo ha operado tradicionalmente con criterios cuyo propósito fundamental ha sido lograr la uniformidad de los escolares en sus modos de ser, pensar y actuar, el tratamiento actual de la diversidad da muestras de importantes cambios en las concepciones y modos de entender lo diverso. Se aboga por una perspectiva inclusiva desde la cual todos han de ser aceptados, dentro y fuera de la escuela, para conseguir una educación sensible a las diferencias.

Por ello, se destaca la importancia de crear lazos, de conectar y desarrollar relaciones interpersonales y redes de apoyo entre todos los miembros de la institución escolar, despertando un sentido de pertenencia y un sentido de comunidad, donde cada persona es un miembro valioso, con derechos y responsabilidades (Arnáiz, 1996).

La diversidad atraviesa los diferentes niveles y componentes del sistema escolar, se manifiesta no solo en los estudiantes, sino también en el claustro de profesores y en la propia escuela como institución única y singular. La escuela se convierte de este modo en un excelente espacio para entrar en contacto con los otros iguales y diferentes, quienes también experimentan reacciones ante la diversidad que uno representa. Esta posibilidad de convivir en la diversidad se acrecienta ante la heterogeneidad estudiantil que caracteriza a los grupos de escolares, e implica aprender a desechar prejuicios y estereotipos negativos que suelen ser con frecuencia la base de la discriminación, así como a superar el temor natural a lo desconocido, a lo que no se nos parece.

Para hacer frente a las diferentes formas de maltrato entre pares en las escuelas, y entre ellas a las prácticas de rechazo y exclusión, se apuesta por el desarrollo de programas orientados al fomento de valores democráticos que promuevan la tolerancia y el respeto a la diversidad y a 
la interculturalidad en los adolescentes y jóvenes, sobre la base de la cooperación multinivel (entre alumnos, entre estos y sus profesores, entre profesores, entre la escuela y otros agentes sociales), y un currículum de la no-violencia desglosado en actividades específicas para su concreción (Díaz-Aguado, 2004). También se proponen talleres dirigidos a los docentes, en aras de generar en ellos un ejercicio reflexivo acerca de las condiciones que participan en la construcción de esquemas de percepción que hacen que estas prácticas de exclusión y discriminación en la escuela se asumen y sean percibidas como naturales, subestimando su importancia. Condiciones que muchas veces involucran los efectos simbólicos derivados del propio discurso pedagógico y del quehacer docente. Es necesario, por tanto, incentivar la conciencia de los agentes escolares, para evitar estos comportamientos y generar espacios libres de violencia que permitan mantener relaciones de convivencia democrática, respetuosa y pacífica (Solís, 2017).

El panorama educativo demanda hoy nuevos modos de asumir lo diverso e invita a abandonar la idea de déficit, desviación o desventaja, para aprender a valorar la diversidad en toda su riqueza. Ello exige, sin duda, profundas transformaciones en los modos de concebir y pensar la escuela, en las políticas, la cultura y las prácticas escolares. La búsqueda de una escuela para todos resulta una necesidad en la sociedad contemporánea, porque, a pesar de la prevalencia de ciertos modelos culturales avalados por la globalización neoliberal, se asiste hoy a la proliferación de nuevos referentes y a una diversificación cada vez mayor de estilos y formas de vida, así como a la reivindicación de otros ya existentes, pero negados e invisibilizados a través de la historia. Consecuentemente, se requiere desarrollar en los jóvenes habilidades sociales que ayuden a fomentar la empatía, la mediación intercultural, la tolerancia y aprender así a reconocer al otro como sujeto portador de derechos individuales para que, en la misma medida, seamos merecedores del reconocimiento de nuestros derechos. El espacio escolar ofrece oportunidades clave en este sentido.

\section{Conclusión}

La exclusión entre pares se manifiesta como parte de las dinámicas relacionales que tienen lugar en la escuela. Estas prácticas de rechazo y exclusión entre los estudiantes se sustentan muchas veces en prejuicios y estereotipos negativos aprehendidos e interiorizados desde la infancia, a través de los procesos de socialización donde la familia y la escuela juegan un papel fundamental. Son formas de exclusión que se expresan no solo en el aislamiento físico, sino por medio de actitudes y acciones deliberadas cuyo propósito es ignorar, silenciar, menospreciar, negar e impedir el ejercicio de los derechos y obligaciones, el reconocimiento y la participación del otro.

Si se entiende la formación como un conjunto de procesos complejos de construcción de significados, sentidos e identidades mediante la interacción, es posible entonces comprender la importancia de la dimensión relacional y el valor de la intersubjetividad, no solo para la cons- 
trucción de conocimientos propios de los procesos de enseñanza-aprendizaje que se desarroIlan en el espacio escolar, sino también, y sobre todo, para la constitución de la identidad personal y social de cada individuo. Urge, por tanto, multiplicar los esfuerzos para atender a lo que sin duda resulta hoy uno de los desafíos prioritarios de la educación: la formación de sujetos capaces de convivir sobre la base del reconocimiento de los derechos del otro y en el respeto a las diferencias.

\section{Referencias}

Arellanos, N., Y. Chirinos, Z. López y L. Sánchez (2007). "Los tipos de maltrato entre iguales". Quaderns Digitals, 1-18. http://www.quadernsdigitals.net/datos/hemeroteca/r 1/ nr 772/a 10404/10404.html

Arias, A. (2015). "Contribución a una teoría crítica de los derechos humanos". En A. Estévez y D. Vázquez (eds.). Derechos humanos y transformación política en contextos de violencia. MéxiCO: FLACSO/UNAM/CISAN, 29-60.

Arnáiz, P. (1996). "Las escuelas son para todos". Siglo Cero, 27(2), 25-34. www.congreso.gob.pe/ comisiones/2006/discapacidad/

Bel, C. (2002). Exclusión social: origen y características. Murcia: Facultad de Letras, Universidad de Murcia. http://enxarxats.intersindical.org/nee/CE exclusio.pdf

Cisneros, I. y M. Cisternas (2007). "Derechos humanos y dignidad de la persona". En Derecho, democracia y no discriminación. México: CONAPRED, 11-24.

Delors, J. (1994). “Los cuatro pilares de la educación". En La educación encierra un tesoro. México: El Correo de la UNESCO, 91-103. http://www.uv.mx/dgdaie/files/2012/11/CPP-DC-DelorsLos-cuatro-pilares.pdf

Díaz-Aguado, M. (2004). Prevención de la violencia y la lucha contra la exclusión: la violencia entre iguales en la escuela y en el ocio. Programa de Intervención y Estudio Experimental. Madrid: INJUVE.

Echeita, G. y M. Sandoval (2002). "Educación inclusiva o educación sin exclusiones". Revista de Educación, 327, 31-48. http://benu.edu.mx/wp-content/uploads/2015/03/Educacion inclusiva o educacion sin exclusiones.pdf

Estévez, E., B. Martínez y T. Jiménez (2009). "Las relaciones sociales en la escuela: El problema del rechazo escolar". Psicología Educativa, 15(1), 45-60. https://www.uv.es/lisis/belen/psicologeduct.pdf

Estrada, O., G. Zárate e I. Izquierdo (2016). "Género, violencia y el discurso del (cyber) bullying en el nivel de educación media superior". Opción, 32(13), 954-978. Maracaibo: Universidad del Zulia. http://www.redalyc.org/articulo.oa?id=31048483045 
Furlán, A. (2012). "Inseguridad y violencia en educación. Problemas y alternativas". Perfiles Educativos, XXXIV, número especial. México: IISUE-UNAM. http://www.scielo.org.mx/pdf/peredu/v34nspe/v34nspea11.pdf

Harris, J. R. (1995). "Where is the Child's Environment? A Group Socialization Theory of Development". Psychological Review, 102(3), 458-489.

Honneth, A. (1997). La lucha por el reconocimiento. Por una gramática moral de los conflictos sociales. Barcelona: Crítica/Grijalbo Mondadori.

Instituto Mexicano de la Juventud - Consejo Nacional para Prevenir la Discriminación. (2011). Encuesta Nacional sobre Discriminación en México. Enadis, 2010. Resultados sobre las y los jóvenes. https://www.conapred.org.mx/userfiles/files/Enadis-JOVENES-Accss.pdf

Kaplún, G. (2003). “El currículum oculto de las nuevas tecnologías". En L. López (coord.). Comunicación Social. Selección de textos. La Habana: Editorial Félix Varela, 198-210.

Lira, L., S. Sandoval y L. Aguilar (2016). Estructuras de convivencia. Una propuesta para la comprensión de la violencia escolar. Jalisco: Red de Posgrados en Educación. https://www. academia.edu/25730471/Estructuras de convivencia. Una propuesta para la comprensi\%C3\%B3n de la violencia escolar

Marc, E. y D. Picard (1992). La interacción social. Cultura, instituciones y comunicación. Barcelona/ Buenos Aires/México: Paidós.

Mead, G. (1982). Espíritu, persona y sociedad. Desde el punto de vista del conductismo social. Barcelona: Paidós Básica.

Meza, L. (2010) La zona de desarrollo próximo (ZDP). https://www.infoamerica.org/documentos word/vygotsky.doc

Organización para la Cooperación y el Desarrollo Económicos (2017). México. Nota sobre el país. Resultados de PISA 2015. Bienestar de los alumnos. https://www.oecd.org/pisa/PISA2015-Students-Well-being-Country-note-Mexico.pdf

Pluma, A. (2011). "Diferentes prismas para estudiar la exclusión social. Marco teórico y propuesta de reconceptualización de la exclusión basado en el sujeto". Documentos de Trabajo Social, 49. https://dialnet.unirioja.es/descarga/articulo/4111404.pdf

Ramírez, A. (2014). “La educación escolar como factor de inclusión social desde un planteamiento sistémico. El caso de la niñez jornalera migrante en los valles de Culiacán, Sinaloa (México)". Tesis Doctoral. Facultad de Pedagogía, Universidad de Barcelona.

Ramos, J. A. (2012). "Cuando se habla de diversidad ¿de qué se habla? Una respuesta desde el sistema educativo". Revista Interamericana de Educación de Adultos, 34(1), 77-96. http:// www.crefal.edu.mx/rieda/images/rieda-2012-1/contrapunto2.pdf

Rizo, M. (2006). “La interacción y la comunicación desde los enfoques de la Psicología Social y la Sociología Fenomenológica. Breve exploración teórica". Anàlisi, 33, 45-62. https://ddd.uab. cat/pub/analisi/02112175n33/02112175n33p45.pdf 
Rodríguez, M. E. (2007). "Los derechos humanos sociales". En Derechos humanos y Desarrollo. Justicia universal: el caso latinoamericano. España: Icaria, 92-103.

Sánchez, F. (2016). "La violencia en las escuelas. El caso de las escuelas secundarias de las zonas metropolitanas de Xalapa y Veracruz, 2008-2012". Tesis Doctoral, Instituto de Investigaciones Histórico-Sociales, Universidad Veracruzana. https://cdigital.uv.mx/bitstream/handle/123456789/47018/SanchezHerreraFrancisco.pdf;jsessionid=80630CBED669F782C792D FA90AF61710?sequence $=1$

Saraví, G. (2015). Juventudes fragmentadas: socialización, clase y cultura en la construcción de la desigualdad. México: FLACSO / CIESAS.

Secretaría de Educación Púbica (2009). Encuesta Nacional sobre Exclusión, Intolerancia y Violencia en las Escuelas de Educación Media Superior. http://bdsocial.inmujeres.gob.mx/index.php/ eneivems-142/encuesta-nacional-de-exclusion-intolerancia-y-violencia-en-escuelas-publicas-de-educacion-media-superior

(2016). Documento Base del Bachillerato General. Subsecretaría de Educación Media Superior. Dirección General del Bachillerato. http://www.dgb.sep.gob.mx/informacion-academica/programas-de-estudio/documentobase/DOC BASE 1605 2016.pdf

Solís, D. (2016). Perspectivas socioculturales sobre exclusión y violencia en la educación. México: Fontamara.

(2017). Procesos educativos en contextos de desigualdad, discriminación, exclusión y violencia. Perspectivas y prácticas desde los y las estudiantes. México: Fontamara.

Tello, N. (2005). "La socialización de la violencia en las escuelas secundarias. Proceso funcional a la descomposición social". Revista Mexicana de Investigación Educativa, 10(27), 1165-1181. México: COMIE. http://www.redalyc.org/pdf/140/14002712.pdf

Valadez, I. (2008). Violencia escolar: maltrato entre iguales en escuelas secundarias de la zona metropolitana de Guadalajara. México: Universidad de Guadalajara-Dirección de Psicopedagogía. http://cvsp.cucs.udg.mx/drupal6/documentos/violencia escolar libro.pdf

Velázquez, L. M. (2005). "Experiencias estudiantiles con la violencia en la escuela". Revista Mexicana de Investigación Educativa, 10(026), 739-764, México: COMIE.

Weiss, E. (2012). "Los estudiantes como jóvenes: el proceso de subjetivación". En Perfiles Educativos, 34(135), 134-148. http://www.iisue.unam.mx/perfiles/perfiles articulo. php?clave $=2012-135-134-148$

(2014). "Subjetivación y formación de la persona". Ponencia en el Congreso Internacional del AFIRSE "Epistemologías y metodologías de la investigación en la educación, México: UNAM. http://departamentos.cinvestav.mx/Portals/die/SiteDocs/Investigadores/EWeiss/Jovenes/ EWJovenes2014-SubjetivacionYformacionDeLaPersona.pdf?ver=2014-05-26-151953-540 Wieviorka, M. (2011). Una sociología para el siglo XXI. Barcelona: UOC Ediciones. 
Yurén, T. (2012). "Fugas autoformativas y resistencia a la exclusión como prácticas de subjetivación". En M. Sánchez y O. Soto (eds.). Interioridad, subjetivación y conflictividad social. México: Universidad Iberoamericana Puebla, 81-96.

Zayas, F. y A. Rodríguez (2010). “Educación y educación escolar”. Actualidades investigativas en Educación, 10(1), 1-21. Costa Rica: Universidad de Costa Rica. 